Original Research Article

\title{
The role of antioxidants and free radicals in the healing effects of Bacopa monniera on acetic acid-induced colitis in rats
}

\author{
Himanshu Sharma ${ }^{1 *}$, Saurabh Chauhan ${ }^{2}$, Punya Pratap Singh ${ }^{3}$, Raj K. Goel ${ }^{4}$
}

${ }^{1}$ Department of Pharmacology,

${ }^{2}$ Department of Surgery,

${ }^{3}$ Department of Radiology,

Bundelkhand Medical College,

Sagar, Madhya Pradesh, India

${ }^{4}$ Department of Pharmacology, Institute of Medical Sciences,

Banaras Hindu University,

Varanasi, Uttar Pradesh, India

Received: 22 July 2017

Accepted: 24 August 2017

*Correspondence to:

Dr. Himanshu Sharma,

Email: himanshugsvm@

gmail.com

Copyright: (C) the author(s), publisher and licensee Medip Academy. This is an openaccess article distributed under the terms of the Creative Commons Attribution NonCommercial License, which permits unrestricted noncommercial use, distribution, and reproduction in any medium, provided the original work is properly cited.

\begin{abstract}
Background: The aim to study and elucidate the healing effects of ethanolic extract of dried whole plant of Bacopa monniera against experimental colitis in rats.

Methods: Bacopa monniera whole plant extract was administered orally, once daily for 14 days, to rats after induction of colitis with acetic acid. We studied its effects on: faecal output, food and water intake, and body weight changes and also examined colonic mucosal damage, inflammation and status of antioxidants: superoxide dismutase, reduced glutathione; free radicals: nitric oxide, lipid peroxidation on 15th day of the experiment. Antibacterial activity of the extract was also studied using in vitro procedures. Statistical comparison was performed using either unpaired ' $t$ ' test or one -way analysis of variance (ANOVA) and for multiple comparisons versus control group was done by Dunnett's test.

Results: Bacopa monniera whole plant extract decreased colonic mucosal damage, inflammation, faecal output and increased body weight in acetic acid induced colitis. It also showed antibacterial activity and enhanced the antioxidant but decreased free radicals. Acute toxicity study indicated no mortality or other ANS or CNS related adverse effects even with ten time effective dose indicating its safety.

Conclusions: Bacopa monniera whole plant extract is safe, effective and could be beneficial as a complementary agent in treatment of ulcerative colitis.
\end{abstract}

Keywords: Antioxidants, Bacopa monniera, Colitis, Free radicals

\section{INTRODUCTION}

Bacopa monniera Linn. or Brahmi (BM) (family: Schropulariaceae) is an annual creeping plant found throughout India in wet, damp and marshy areas. In Indian traditional literature, it is categorized as a Medhyarasayana having sedative, tranquilizing, antioxidant, anti-inflammatory, and memory-enhancing effects. ${ }^{1,2}$ It improves the performance of rats in various learning situations, in motor learning, and reverse anterograde amnesia induced by scopolamine and sodium nitrite in mice. ${ }^{3} \mathrm{BM}$ is a potent free radical scavenger (antioxidant) that reduces $\mathrm{H}_{2} \mathrm{O}_{2}$ induced cytotoxicity and DNA damage in human fibroblast. ${ }^{4}$ It contains, apart from other saponins, bacoside $\mathrm{A}$, which is the major chemical moiety responsible for pharmacological effects of the drug. ${ }^{5,6}$ Previous work in our department establishes its antioxidant properties in gastric ulcer models. ${ }^{7}$ Due to antioxidant properties, BM is used for the treatment of asthma, epilepsy, insanity, inflammatory and cardiovascular diseases. ${ }^{8,9}$ Therefore, in view of the activities performed by this plant, investigation must be continued in the recently observed actions. 
Ulcerative colitis (UC), a subcategory of inflammatory bowel disease, is a refractory, chronic, and nonspecific disease of colon. It involves only the innermost lining or mucosa and manifests as continuous area of inflammation and ulceration, with no segment of normal tissue. Symptoms include progressive diarrhea with urgency, rectal bleed, colicky abdominal pain, weight loss, fever, and fatigue. It is diagnosed prior to age of 30 and affect men and women equally. It is more frequent in Western countries, specifically Northern Europe and North America; however, increases are now reported from Asia, Africa and Latin America. ${ }^{10}$ The classical therapeutic strategies aim to control the exacerbated host immune response with 5-aminosalicylates (5-ASA) and corticosteroids; immunomodulators (such as, azathioprine and 6-mercaptopurine) and antibiotics are used in steroid resistant and steroid-dependent patients. ${ }^{11}$ However, all of these drugs have shortcomings.

Although the exact cause remains undetermined, the condition is related to genetic, immunological and environmental factors. Recently, altered oxidant/antioxidant status in the inflamed colon has received attention in both humans and animals. Reactive oxygen species (ROS) are produced in excess by the inflamed mucosa and may be pathogenic. ${ }^{12}$ The main source of ROS is activated leukocytes and neutrophils which impair cell membrane stability and cause cell death by lipid peroxidation. ${ }^{13}$ These are controlled by cellular defence comprising enzymatic (i.e., superoxide dismutase and catalase) and non-enzymatic (reduced glutathione) scavengers.

The use of herbal therapies for UC is increasing because of a lack of safety and efficacy of standard drugs. ${ }^{14}$ In view of the reported antioxidant, anti-inflammatory and ulcer protective properties of $B$. monniera, the present work investigates effects of the plant extract on experimental colitis in rats and attempts to elucidate the mechanism/s of its healing effects by analyzing the role of oxidative stress generated and protective effect, on tissue antioxidant defense system and lipid peroxidative status.

\section{METHODS}

\section{Animals}

The study was conducted using inbred Charles-Foster albino rats $(150-250 \mathrm{~g})$ and mice $(25-30 \mathrm{~g})$ of either sex housed in colony cages at ambient temperature of $26 \pm 2{ }^{\circ} \mathrm{C}$ and $44-56 \%$ relative humidity, with light and dark cycles of 10 and $14 \mathrm{~h}$ respectively for 1 week before and during the experiments. Animals were provided with standard rodent pellet diet (Pashu Aahar, Ramnagar, Varanasi) and the food was withdrawn 18/24 h before the experiment though water was allowed ad libitum. 'Principles of laboratory animal care' (NIH publication no. 82-23, revised 1985) guidelines were followed.

\section{Extraction and standardization of Bacopa monniera}

\section{Preparation of extract}

Whole plant of B. monniera was collected in September from Ayurvedic Garden, and identified with the standard sample preserved in the department of Dravyaguna, Institute of Medical Sciences, Varanasi. They were dried in shade, pulverized and extraction was done with $50 \%$ ethanol. The procedure was repeated twice and the $50 \%$ ethanolic extract (BME) was filtered and dried at $40^{\circ} \mathrm{C}$ in incubator and stored at $-20^{\circ} \mathrm{C}$. The yield was about $28.16 \%$ $(\mathrm{w} / \mathrm{w})$.

\section{Standard drug}

Sulfasalazine (SS; SAZO, $1000 \mathrm{mg}$ tablet, WALLACE); acetic acid obtained from Merck Ltd., Mumbai, India, and all the other chemicals and reagents were used of analytical grade.

\section{Experimental procedure}

\section{Induction of colitis in rats and treatment protocol}

Colitis in rats was induced by intra-colonic application of $0.2 \mathrm{ml}$ of $10 \%$ acetic acid (AA) given perrectally. Control group received intracolonic normal saline (NS-control).

BME and SS, suspended in $1 \%$ carboxy methyl cellulose (CMC) in distilled water were given once daily for a period of 14 days after induction of colitis: control group received $1 \%$ CMC only. Healing effects of graded doses of BME $(12.5,25$ and $50 \mathrm{mg} / \mathrm{kg})$ were studied initially for a period of 14 days on colonic mucosal weight, damage score and adhesions. For further studies, an optimal dose of $25 \mathrm{mg} / \mathrm{kg}$ was chosen from the dose response curve. The effects of BME was seen on various (a) physical parameters like food and water intake, diarrhea with or without mucus/blood and body weight changes; colonic damage score, adhesions, mucosal weight, and macroscopic/microscopic pathological changes in the colonic tissue and (b) biochemical parameters like free radicals (lipid peroxidation; LPO and nitric oxide; NO) and antioxidants (enzymatic, superoxide dismutase; SOD and nonenzymatic, reduced glutathione; GSH) in the colonic tissue after 14 days of treatment. The results were compared with standard drug for UC (SS, $100 \mathrm{mg} / \mathrm{kg}$ ).

\section{Acute toxicity study in mice}

An acute toxicity study was done following OECD guidelines 420 (OECD 2000). Overnight fasted, healthy mice weighing 20 to 25 gm were administered BME orally in a dose of up to 10 times of optimal effective dose. Animals were observed closely for first four hours, for any toxic manifestations like increased motor activity, salivation, convulsion, coma and death. Subsequently observations were made at regular intervals for any ANS, 
CNS activities and mortality for $24 \mathrm{hr}$ and further up to a period of 14 days.

Assessment of faecal output and changes in body weight, food intake and water intake

All parameters measured on the 0, 2, 4, 6, 10 and 14th day of the experiment and the results were compared. $24 \mathrm{hr}$ stool frequency and mucous or blood stained diarrhea of individual rat were observed. Each rat was weighed on the standard weighing machine. A measured weight of food was given to each rat in the respective cages at a fixed time (10:00 am morning) and next day the amount of food left was weighed again. Similarly, a measured volume of water was given to each rat at a fixed time in bottles and next day measured with help of measuring cylinder.

\section{Assessment of damage and inflammation}

All scorings of damage were done by observer unaware of the treatment group. The rats were randomized and weighed before sacrifice, by an overdose of ether, and proximal $8 \mathrm{~cm}$ of colon was removed. The colon was opened by a longitudinal incision and macroscopically visible damage was scored on 0-10 scale, based on area involved and the presence or absence of ulcers, as described by Morris et al, 1989. For histology samples were fixed in $10 \%$ formalin, embedded in paraffin and stained with hematoxylin-eosin. Tissue samples were taken for measurement of weight, and biochemical estimation of antioxidants- SOD and GSH and free radicalLPO and NO.

Estimation of colonic mucosal antioxidants and free radicals

Protein content in the colonic mucosal homogenate was estimated following the procedure of Lowry et al. ${ }^{15}$ Lipid peroxidation (LPO) product malondialdehyde was estimated using 1,1,3,3- tetraethoxypropane as standard and was expressed as nmol/g wet tissue. ${ }^{16}$ Nitric oxide (NO) levels were estimated by the Griess reaction method and result was expressed as nmol/g wet tissue. ${ }^{17}$ The inhibition of reduction of nitro blue tetrazolium (NBT) to blue colored formozan in presence of phenazine metha sulphate (PMS) and NADH was measured at $560 \mathrm{~nm}$ using n-butanol as blank for Superoxide dismutase (SOD). ${ }^{18}$ Reduced glutathione (GSH) was measured in the colonic mucosal homogenate scrap following the standard procedure of Sedlak and Lindsay. ${ }^{19}$ SOD was expressed as $\mathrm{mU} / \mathrm{mg}$ protein while NO, SOD, GSH expressed as nmol/g protien.

Antimicrobial susceptibility and minimum inhibitory concentration (MIC)

The antimicrobial activity of the extract was evaluated using disc diffusion method against four bacterial strains: Escherichia coli ATCC 25922, Shigella boydii, Shigella sonnei and Shigella flexneri. ${ }^{20}$ Diameters of the zone of inhibition (in $\mathrm{mm}$ ) were measured. MIC was determined by micro-dilution method using serially diluted ( 2 folds) plant extracts according to the National Committee for Clinical Laboratory Standards, 2000 (NCCLS). ${ }^{21}$

\section{Statistical analysis}

All data expressed as mean \pm standard error of the mean. The one -way analysis of variance (ANOVA), followed by the Dunnett's multiple comparison tests, was performed. All statistical analysis was done using SPSS statistical version 16.0 software package (SPSS ${ }^{\circ}$ Inc., USA). A probability value of $<0.05$ was accepted as statistically significant difference between the vehicle-treated control and drug treated groups.

\section{RESULTS}

\section{Effects on colonic damage, inflammation and adhesions}

AA induced colitis led to significant increase in colonic mucosal damage score $(6.16 \pm 0.54, \mathrm{P}<0.001)$, adhesions $(83.3 \%)$ and increase in colonic weight to $227.6 \pm 17.3$ (56.6\%) compared with NS group indicating an extensive colonic tissue damage, inflammation together with adhesions (Table 1). BME given in graded doses of 12.5, 25 and $50 \mathrm{mg} / \mathrm{kg}$ showed dose-dependent decrease in damage score from $39.6 \%$ to $67.9 \%$ ( $\mathrm{P}<0.05$ to $\mathrm{P}<0.001$ ), colonic weight from $17.7 \%$ to $30.4 \%(\mathrm{P}<0.01)$ and tissue adhesions from (40\% to $80 \%)$ and the effects were comparable with sulfasalazine. From the above dose response study, BME $(25 \mathrm{mg} / \mathrm{kg})$ showing optimal healing effects were selected for future studies.

Table 1: Effects of graded doses BME on AA- induced changes in rat colonic mucosal damage score, weight and adhesions.

\begin{tabular}{|llll|}
\hline $\begin{array}{l}\text { Oral } \\
\text { treatment } \\
(\mathbf{m g} / \mathrm{kg}, \\
\text { OD x 14 } \\
\text { days })\end{array}$ & $\begin{array}{l}\text { Damage } \\
\text { score } \\
(\mathbf{0}-10)\end{array}$ & $\begin{array}{l}\text { Colonic } \\
\text { Weight } \\
(\mathrm{mg} / \mathrm{cm})\end{array}$ & $\begin{array}{l}\text { Adhesions } \\
(\%)\end{array}$ \\
\hline $\begin{array}{l}\text { NS+CMC } \\
(1 \%)\end{array}$ & 0.0 & $145.3 \pm 8.20$ & 0.0 \\
\hline $\begin{array}{l}\text { AA+CMC } \\
(1 \%)\end{array}$ & $6.16 \pm 0.54^{* * *}$ & $227.6 \pm 17.3^{* * *}$ & 83.33 \\
\hline $\begin{array}{l}\text { AA+BME } \\
(12.5)\end{array}$ & $3.72 \pm 0.61^{\mathrm{a}}$ & $187.3 \pm 11.7$ & 50.00 \\
\hline $\begin{array}{l}\text { AA+BME } \\
(25)\end{array}$ & $2.00 \pm 0.68^{\mathrm{c}}$ & $165.5 \pm 12.6^{\mathrm{b}}$ & 33.33 \\
\hline $\begin{array}{l}\mathrm{AA}+\mathrm{BME} \\
(50)\end{array}$ & $1.98 \pm 0.57^{\mathrm{c}}$ & $158.3 \pm 10.1^{\mathrm{b}}$ & 16.67 \\
\hline
\end{tabular}

Results are mean \pm SEM of 6 rats in each group. Values in parenthesis indicate $\%$ of respective AA group

$* * *<0.001$ compared to respective NS group (unpaired ' $t$ ' test),

a $<0.05$, b $<0.01,{ }^{c}<0.001$, compared to respective AA group (Statistical analysis was done by one way analysis of variance followed by Dunnett's test for multiple comparisons).

[NS: Normal saline; AA: Acetic Acid; BME: Bacopa monniera Extract; SS: Sulfasalazine; CMC: carboxymethyl cellulose] 


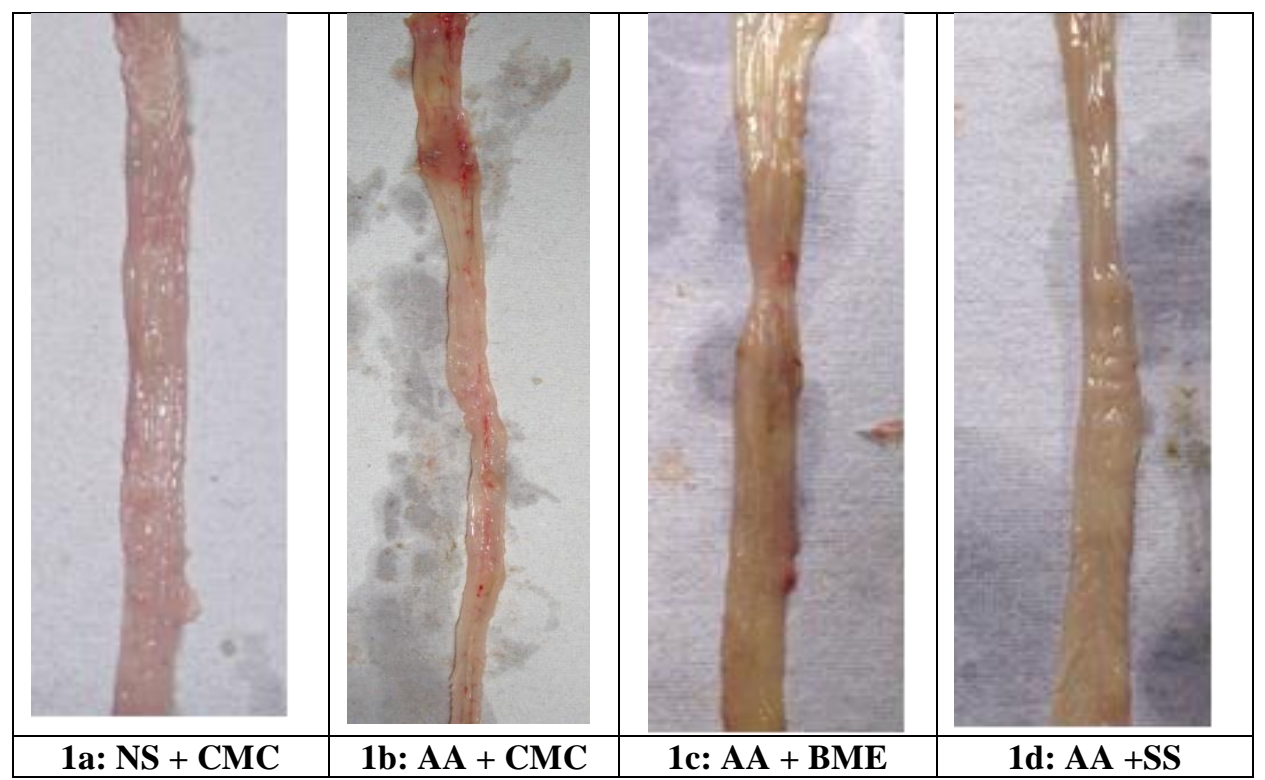

[NS: Normal saline; AA: Acetic Acid; BME: Bacopa monniera Extract; SS: Sulfasalazine; CMC: carboxymethyl cellulose]

Figure 1a: Macroscopic changes seen in saline enema treated colon of CMC-treated rat showing normal morphology. Figure 1b: Macroscopic changes seen in the colon of AA-induced colitis rat treated with CMC showing hydropsia, necrosis, erosion and ulceration. Figure 1c and 1d: Macroscopic changes seen in the colon of AA-induced colitis rat treated with BME $(25 \mathrm{mg} / \mathrm{kg})$ and SS $(100 \mathrm{mg} / \mathrm{kg})$ showing reduction in ulceration, erosion, necrosis and hydropsia

Figure 1: AA-induced colitis macroscopic study.

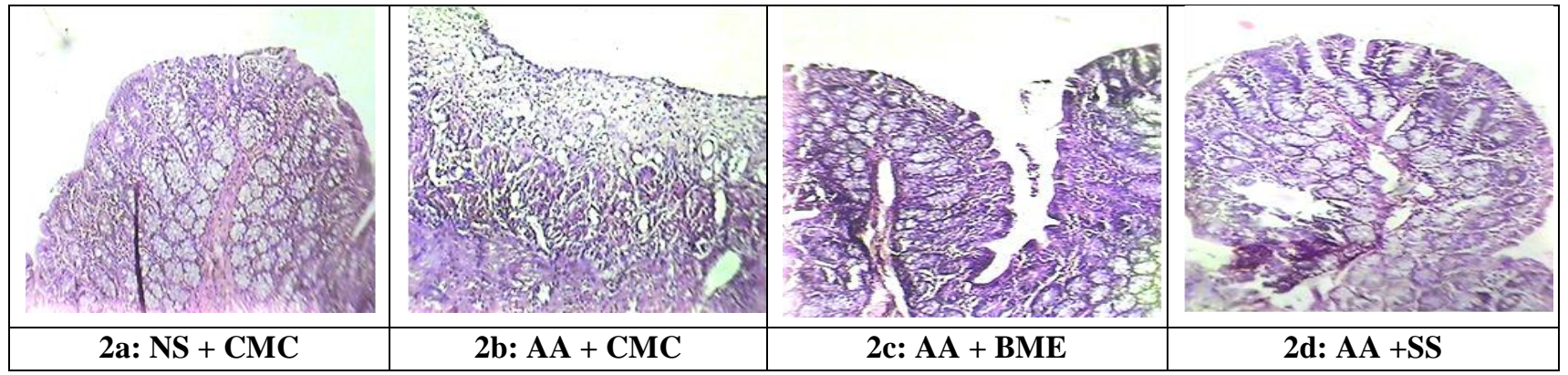

[NS: Normal saline; AA: Acetic Acid; BME: Bacopa monniera Extract; SS: Sulfasalazine; CMC: carboxymethyl cellulose]

Figure 2a: The photomicrographs of saline enema treated colon of rats treated orally with CMC. The structure is relatively normal and clear with intact epithelia, normal glands and abundant goblet cells except a small amount of inflammatory cells infiltration in the mucosa. Figure 2b: The photomicrographs of the AA-treated colon rat treated orally with CMC. The cryptae formations are deformed and the epithelia are not intact. There are distorted glands, loss of goblet cells, and lymphocytic infiltration in mucous layer and edema in submucosa of the colon. Figure $2 \mathrm{c}$ and $2 \mathrm{~d}$ : The photomicrographs of the AA-treated colon of rat treated orally with either BME or PE or SS. The treatments with the extracts/drug show improvement in the structures with near intact epithelia and normal glands. The infiltration of lymphocytes is decreased but still visible.

Figure 2: AA-induced colitis microscopic study.

\section{Histopathology study}

Macroscopic study

The severity of hydropsia, necrosis, erosion and ulceration in the colon of AA-treated rats (Figure 1b) significantly reduced after administration of BME or SS (Figure 1c and 1d).

\section{Microscopic study}

The cryptae are deformed and the epithelia is damaged alongwith distorted glands, loss of goblet cells, and 
lymphocytic infiltration in mucosa and edema in submucosa in AA-treated colon. Protective effects of BME and SS shown by improvement in the structures with near intact epithelia and normal glands. The infiltration of lymphocytes decreased but still visible (Figure 2).

Effects on diarrhea, fecal output and presence of blood or mucous

AA treated rats showed gradual increase in fecal output from day 2 onwards (129\%) and at day 14 it was $130.2 \%$ compared with Day 0 frequency and was associated with presence of blood till day 6 and mucous till day 10. BME administration showed decrease in stool frequency from day 4 onwards and decrease in duration of blood/mucous in the stool which was comparable with sulfasalazine (100mg/kg) (Figure 3).

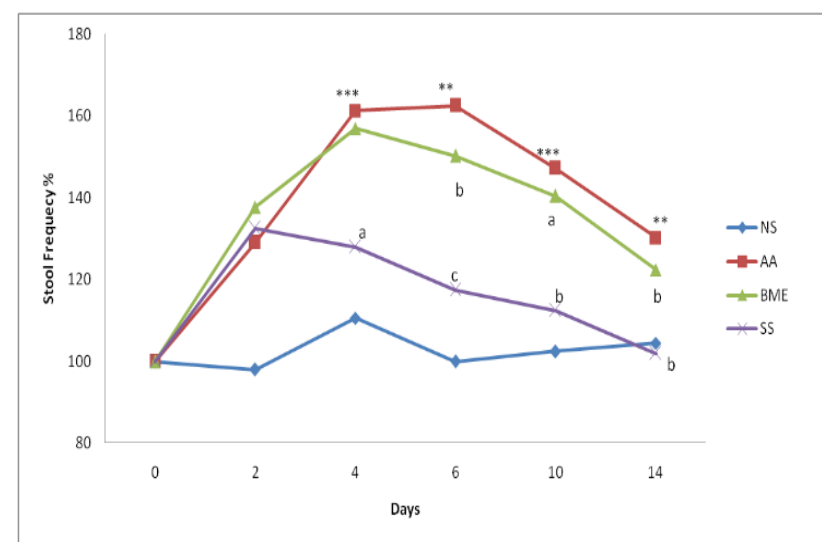

Results are mean \pm SEM of 6 rats in each group.

$* *<0.01, \quad * * *<0.001$ compared to respective NS group (unpaired 't' test), ${ }^{\mathrm{a}<0.05},{ }^{\mathrm{b}}<0.01,{ }^{\mathrm{c}}<0.001$ compared to respective AA group (Statistical analysis was done by one way analysis of variance followed by Dunnett's test for multiple comparisons). [NS: Normal saline; AA: Aetic Acid; BME: Bacopa monniera Extract; SS: Sulfasalazine]

Figure 3: The effects of BME $(25.0 \mathrm{mg} / \mathrm{kg})$ and SS $(100 \mathrm{mg} / \mathrm{kg})$ on rat stool frequency $(\%)$ in AA- induced colitis.

Effects on body weight changes and food and water intakes
AA-induced colitis led to gradual decrease in body weight as observed from day 2 onwards till $14^{\text {th }}$ day of study. Treatment with BME reversed the decrease trend in body weight suggestive of beneficial effects of the test extracts and the result was comparable with sulfasalazine. However, little or no change was observed on food and water intake between the AA-treated and BME and SS treated animals from 0 day to $14^{\text {th }}$ day of study treatments (Figure 4).

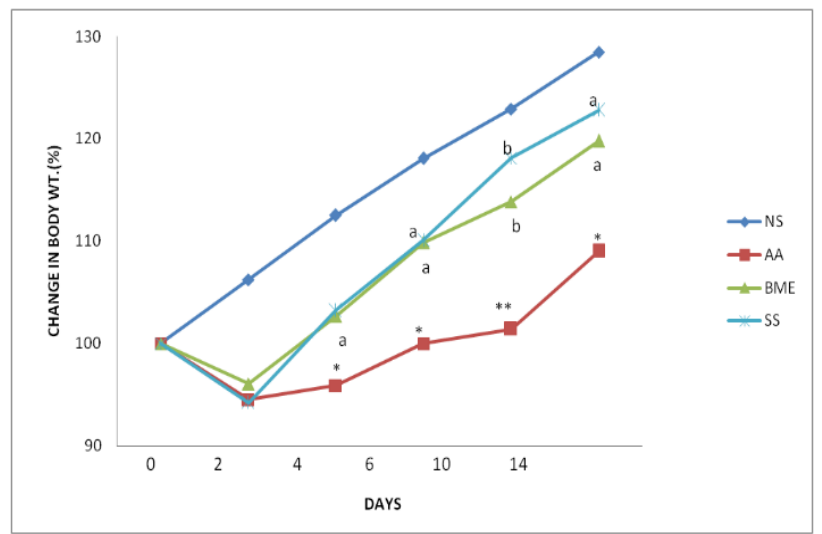

Results are mean \pm SEM of 6 rats in each group.

$*<0.05, * *<0.01$ compared to respective NS group (unpaired ' $t$ ' test), ${ }^{a}<0.05, \quad b<0.01$, compared to respective AA group (Statistical analysis was one by one way analysis of variance followed by Dunnett's test for multiple comparisons).

[NS: Normal saline; AA: Acetic Acid; BME: Bacopa monniera Extract; SS: Sulfasalazine]

Figure 4: The effects of BME $(25.0 \mathrm{mg} / \mathrm{kg})$ and SS $(100 \mathrm{mg} / \mathrm{kg})$ on change in rat body weight $(\%)$ in AAinduced colitis.

\section{Effects on colonic mucosal free radicals and antioxidant status}

\section{Effects on free radicals}

Oxidative free radical (LPO and NO) were enhanced in AA acid induced colitis group. BME showed decrease in levels of both LPO $(41.7 \%, \mathrm{p}<0.001)$ and NO $(34.6 \%$, $\mathrm{p}<0.001)$ near to control group and the effect was comparable with SS (Table 2).

Table 2: Effects of BME and SS treatment on AA-induced changes in Free Radicals (lipid peroxidation, LPO and nitric oxide, NO) and Antioxidants (superoxide dismutase, SOD and glutathione, GSH) in rat colonic mucosa.

\begin{tabular}{|c|c|c|c|c|}
\hline \multirow{3}{*}{$\begin{array}{l}\text { Oral treatment } \mathrm{mg} / \mathrm{kg} \text {, once } \\
\text { daily } x 14 \text { days) }\end{array}$} & \multicolumn{4}{|c|}{ Anti-oxidants } \\
\hline & LPO & NO & SOD & GSH \\
\hline & nmol/mg protein & nmol/mg protein & IU/mg protein & nmol/mg protein \\
\hline $\mathrm{NS}+\mathrm{CMC}(1 \%)$ & $6.3 \pm 0.38$ & $13.0 \pm 1.12$ & $0.39 \pm 0.04$ & $27.5 \pm 2.77$ \\
\hline $\mathrm{AA}+\mathrm{CMC}(1 \%)$ & $15.8^{* * *} \pm 1.22$ & $24.3^{* * *} \pm 2.36$ & $0.16^{* * *} \pm 0.03$ & $15.5^{* *} \pm 1.29$ \\
\hline AA+BME (25) & $6.6^{c} \pm 0.45$ & $8.4^{c} \pm 1.29$ & $0.40^{c} \pm 0.03$ & $29.9^{c} \pm 2.48$ \\
\hline $\mathrm{AA}+\mathrm{SS}(100)$ & $6.6^{c} \pm 0.59$ & $8.3^{c} \pm 0.29$ & $0.31^{b} \pm 0.01$ & $30.0^{\mathrm{c}} \pm 2.18$ \\
\hline
\end{tabular}

Results are mean \pm SEM of 6 rats in each group. $* *<0.01, * * *<0.001$ compared to respective NS group (unpaired ' $t$ ' test),

$\mathrm{b}<0.01,{ }^{\mathrm{c}}<0.001$, compared to respective AA group (Statistical analysis was done by one way analysis of variance followed by Dunnett's test for multiple comparisons).

[NS: Normal saline; AA: Acetic Acid; BME: Bacopa monniera Extract; SS: Sulfasalazine; CMC: carboxymethyl cellulose; LPO: Lipid peroxide; NO: Nitric Oxide; SOD: Superoxide dismutase; GSH: Reduced glutathione] 
Effect on antioxidants (SOD and NO)

AA treated animals showed significant decrease in both SOD and GSH levels in the colonic mucosal incubates compared to normal untreated rats. BME and SS reversed the above change in SOD $(250 \%, \mathrm{p}<0.001)$ and $\mathrm{GSH}$ $(193 \%, \mathrm{p}<0.001)$ levels near to NS group (Table 2).

\section{In-vitro antimicrobial test}

Showed the susceptibility test with BME against gram negative bacteria viz. Escherichia coli ATCC 25922, Shigella boydii, Shigella sonnei and Shigella flexneri.

\section{Acute toxicity study}

Neither mortality nor any visible changes were observed during the acute toxicity studies with dose of BME up to $250 \mathrm{mg} / \mathrm{kg}$ (10 times optimal dose) and hence was found to be safe in laboratory animals.

\section{DISCUSSION}

Ulcerative colitis (UC), a nonspecific inflammatory lifelong disease of the large intestine, cause profound emotional and social impacts and may progress to colon cancer. ${ }^{22,23}$ Although it is treated with anti-inflammatory or immunosuppressive drugs, most of these prove to be inadequate. Consequently, many patients turn to alternative traditional plant based remedies. Bacopa monniera (Brahmi Rasayan) a medicinal herb, mainly used to improve memory and intellect, is efficacious in alleviating inflammation. An ethanolic extract of BM reduce lipid peroxidation and promotes antioxidant status. $^{24}$

Based on ethnopharmacological uses, we investigated the anti-inflammatory effects of BM ethanol extract in an in vivo model of UC induced by AA in CF rats. This model exhibits symptoms comparable to those of human ulcerative colitis, such as: weight loss, diarrhea, bloody faeces, mucosal ulceration and also serves as a model to investigate the role of free radicals and antioxidants in the pathophysiology of colitis. ${ }^{25}$

We administered BME orally and evaluated its therapeutic effects after AA induced colitis. Our findings demonstrate that BME significantly suppresses colitis in rats, and improves body weight, stool frequency, and decreases intestinal bleeding. In the present study, Sulfasalazine (5ASA/SS) was used as a reference drug. It is structurally related to nonsteroidal anti-inflammatory drugs (NSAIDs), such as, aspirin, and targets inflammation, proliferation, and apoptosis. BME administration was also found to attenuate increase in colonic mucosal damage score and adhesions (indicative of necrosis and ulcerations) and increase in colonic weight (indicative of inflammation and hydropsia) induced by AA, and markedly reduce neutrophil and macrophage. Histopathology findings were in line with our physical findings, viz. - colons of rats treated with AA presented inflammatory cell infiltration, mucosal erosion, and crypts distortion. BME/SS reduced all inflammatory changes.

Oxidative stress is believed to be a major cause of tissue destruction in UC and involves reactive oxygen and nitrogen metabolites. In our experiment, BME diminished free radicals (NO and LPO) and increased antioxidants (SOD and GSH) in the colonic tissues of AA treated rat (Table 2) which provides a clue as to how BME has an anti-inflammatory effect. Moreover the antimicrobial potential of BME was also established indicating it to be a contributory factor.

Summarizing, the study suggests potent anti-inflammatory effects of BME in AA-induced colitis mediated via the inhibition of free radical and increase in antioxidants effectively counteracting the free radicals as well as the antimicrobial effect. Furthermore, BME $(25 \mathrm{mg} / \mathrm{kg})$ was found to have therapeutic effects comparable to SS $(100 \mathrm{mg} / \mathrm{kg})$, which is currently used to treat UC. These results strongly suggest that the modulation of pathophysiological activity by BME offers a promising means for the treatment of diseases characterized by inflammation of the gastrointestinal tract. However, further study is required to isolate the phytochemicals in BME responsible for these activities and to elucidate the mechanism responsible for its therapeutic effects.

\section{ACKNOWLEDGEMENTS}

The authors' express sincere thanks to Department of Pharmacology, Faculty of Medicine, Institute of Medical Sciences, Banaras Hindu University, Varanasi, India, for providing the best facilities and funding for the research work.

\section{Funding: No funding sources \\ Conflict of interest: None declared}

Ethical approval: The study was approved by the Institutional Animal Ethics Committee (Dean/10-11/276 dated 13.10.2010)

\section{REFERENCES}

1. Udupa KN, Singh RH. Clinical and Experimental Studies on Rasayana drugs and Panchkarma Therapy, Central Council for Research in Ayurveda and Siddha. New Delhi; 1995.

2. Shabana CA, Muhammad DSA, Atta-ur-Rahman Y. Anti-inflammatory activity of Bacopa monniera in rodents. J Ethnopharmacol. 2006;104:286-9.

3. Kishore K, Singh M. Effect of bacosides, alcoholic extract of Bacopa monniera Linn. (Brahmi), on experimental amnesia in mice. Indian $\mathrm{J}$ Exp Biol. 2005;43:640-5.

4. Russo A, Angelo AI, Francesca B, Marcella R, Angelo V. Free radical scavenging capacity and protective effect of Bacopa monniera L. on DNA damage. Phytother Res. 2003;17:870-5. 
5. Garai S, Mahato SB, Ohtani K, Yamasaki K. Dammarane- type Triterpenoid saponins from B. monniera. Phytochemistry. 1996;42:815-20.

6. Sairam K, Dorababu M, Goel RK, Bhattacharya SK. Antidepressant activity of standardized extract of Bacopa monniera in experimental models of depression in rats. Phytomedicine. 2002;9:207-11.

7. Sairam K, Rao Ch V, Dora Babu M, Goel RK. Prophylactic and curative effects of Bacopa monniera in gastric ulcer models. Phytomedicine 2001;8:42330 .

8. Bhattacharya SK, Bhattacharya A, Kumar A, Ghosal S. Antioxidant activity of B. monniera in rat frontal cortex, striatum, and hippocampus. Phytotherapy Research. 2000;14:174-79.

9. Vijayan V, Helen A. Protective activity of Bacopa monniera Linn on nicotine induced toxicity in mice. Phytotherapy Research. 2007;21:378-81.

10. Hanauer SB. Inflammatory bowel disease: epidemiology. Pathogenesis and Therapeutic Opportunities. 2006;12:S3-9.

11. Hanauer S, Sninsky CA, Robinson M. An oral preparation of mesalamine as long-term maintenance therapy for ulcerative colitis. Ann Intern Med. 1996;124:204-11.

12. Keshavarzian A, Morgan G, Sedghi S, Gordon JH, Doria M. Role of reactive oxygen metabolites in experimental colitis. Gut. 1990;31:786-90.

13. Grisham MB, Granger DN. Neutrophil-mediated mucosal injury. Role of reactive oxygen metabolites. Dig Dis Sci. 1988;33:6S-15S.

14. Rahimi R, Mozaffari S, Abdollahi M. On the Use of Herbal Medicines in Management of Inflammatory Bowel Diseases: A Systematic Review of Animal and Human Studies. Dig Dis Sci. 2009;54:471-80.

15. Lowry OH, Rosenborough NJ, Farr AL, Randal RJ. Protein measurement with folin phenol reagent. J Biol Chem. 1951;193:265-75.
16. Okhawa H, Ohishi N, Yagi K. Assay for lipid peroxides in animal tissues by thiobarbituric acid reaction. Anal Biochem. 1979;95:351-8.

17. Miranda KM, Epsey MG, Wink DA. A rapid, simple spectophotometric method for simultaneous detection of nitrate and nitrite. Biol Chem. 2001;5:62-71.

18. Kakkar P, Das B, Viswanathan PN. A modified spectrophotometric assay of superoxide dismutase, Indian J Biochem Biophy. 1984;21:130.

19. Sedlak J, Lindsay RH. Estimation of total protein bound and nonprotien sulfhydryl groups in tissue with Ellman's reagent. Anal Biochem. 1968;25:192.

20. Singh RK, Nath G. Antimicrobial activity of Elaeocarpus sphaericus. Phytother Res. 1999;13:44850.

21. Irith W, Kai H, Robert EW H. Agar and broth dilution methods to determine the minimal inhibitory concentration (MIC) of antimicrobial substances. Nature Protocols. 2008;3:163-75.

22. Asakura H. Ulcerative colitis and intestinal bleeding. Nippon Rinsho. 1998;56:2354-9.

23. Shanahan F, Bernstein CN. The evolving epidemiology of inflammatory bowel disease. Current Opinion in Gastroenterology. 2009;25:301-5.

24. Channa S, Dar A, Anjum S, Yaqoob M, Rahman A. Anti-inflammatory activity of Bacopa monniera in rodents. Journal of Ethnopharmacology. 2006;104:286-9.

25. Pfeiffer CJ, Qiu BS. Effects of chronic nitric oxide synthase inhibition on TNB-induced colitis in rats. J. Pharm. Pharmacol. 1995;47:827-32.

Cite this article as: Sharma $\mathrm{H}$, Chauhan $\mathrm{S}$, Singh PP, Goel RK. The role of antioxidants and free radicals in the healing effects of Bacopa monniera on acetic acid-induced colitis in rats. Int J Basic Clin Pharmacol 2017;6:2388-94. 